\title{
La calidad de los contenidos educativos reutilizables: diseño, usabilidad y prácticas de uso ${ }^{i}$
} \author{
practices of use \\ Teresa Mauri \\ Universidad de Barcelona. Barcelona, España \\ teresamauri@ub.edu \\ Javier Onrubia \\ Universidad de Barcelona. Barcelona, España \\ javier.onrubia@ub.edu \\ César Coll \\ Universidad de Barcelona. Barcelona, España \\ ccoll@ub.edu \\ Rosa Colomina \\ . Universidad de Barcelona. Barcelona, España \\ rosacolomina@ub.edu
}

Analysing the quality of reusable learning objects: design, usability and

\section{Resumen}

El escrito aborda el tema del análisis de la calidad educativa de los contenidos educativos reutilizables desde la perspectiva constructivista de orientación sociocultural del proceso de enseñanza y de aprendizaje. Basándose en este planteamiento y con la finalidad de delimitar la naturaleza de las propuestas formativas cuya calidad pretende ser analizada, define las características específicas de las que integran este tipo de contenidos $\mathrm{y}$, finalmente, plantea algunas propuestas sobre las dimensiones más relevantes a abordar en dicho análisis y sobre la multiplicidad de perspectivas a considerar para poder llevarlo a cabo con garantías de éxito.

Palabras clave: objetos de aprendizaje, entornos virtuales de enseñanza y aprendizaje, calidad en el aprendizaje virtual, diseño instruccional, uso educativo de las TIC, interactividad, constructivismo sociocultural.

\begin{abstract}
The aim of this paper is to discuss the question of reusable learning objects'quality. We depart from a socio-constructivist instructional framework. It is proposed that, when analyzing the quality of learning objects, we must take into account several related dimensions: on the one hand, the technological and pedagogical design of the learning environments in which learning objects are included; on the other hand, the actual uses of these objects by the participants in the teaching-learning process.
\end{abstract}

Keywords: learning objects, virtual learning environments, quality in virtual learning, instructional design, educational uses of ICT, joint activity, socio-constructivism.

\section{Introducción}

El principal objetivo de estas páginas es plantear algunas dimensiones para el análisis de la calidad educativa de propuestas formativas que integran contenidos educativos reutilizables (objetos de aprendizaje). 
Los denominados contenidos educativos reutilizables remiten a una propuesta educativa centrada en los contenidos de aprendizaje que se presenta en formato multimedia o hipermedia y cuya utilización se prevé que sea posible en situaciones en que el grado de coincidencia física, espacial y temporal de profesores y alumnos pueda darse en un grado mínimo o incluso no existir. Este tipo de propuestas han tenido, en muchos casos, y con los riesgos que supone cualquier generalización, algunas características peculiares, que a su vez conllevan consecuencias de especial importancia para el análisis de la calidad de los contenidos a los que nos hemos referido:

a) Son propuestas que, en términos del "triángulo interactivo" básico que define los procesos formales de enseñanza y aprendizaje (el triángulo formado por las relaciones mutuas entre el alumno que aprende, el contenido a aprender y el profesor que guía y orienta el aprendizaje), se centran de manera fundamental en la consideración de los contenidos de aprendizaje, de la actividad mental cognitiva del alumno, o de la relación entre ambos, es decir, en las posibilidades que la propuesta ofrece al alumno de desarrollar una actividad mental constructiva para apropiarse de los contenidos y construir conocimiento; el polo del profesor, o de la ayuda educativa a esa construcción, aparece, sin embargo, y típicamente, en un segundo plano.

b) Se centran en el diseño de la propuesta de contenidos educativos, dejando en un segundo plano los aspectos relativos al desarrollo del proceso de enseñanza y aprendizaje basado en el uso de dichos contenidos.

c) Toman en consideración, en muchos casos, los aspectos técnicos o los aspectos pedagógicos de manera aislada, pero sin profundizar en la toma en consideración de las relaciones entre ambos, que reconvierten la propuesta técnica $o$ pedagógica en otra cualitativamente diferente en cada caso.

d) Del mismo modo, y también en muchos casos, las propuestas de contenidos educativos reutilizables se diseñan con relativa independencia del entorno educativo en que se inscribe y que modifica el alcance de las posibilidades educativas de dichos materiales. En relación a valorar la calidad educativa no puede ser lo mismo que la propuesta de contenidos se inscriba en un entorno educativo de autoaprendizaje, en un entorno educativo semipresencial o en un entorno educativo virtual, ni que se lleven a cabo en el marco de entornos virtuales de unas características u otras, con unas herramientas u otras, con una estructura u otra.

A nuestro juicio, y en el marco anterior, es necesario y urgente plantear una aproximación al análisis de la calidad de las propuestas educativas apoyadas en contenidos educativos reutilizables que contemple la complejidad del proceso de enseñanza y aprendizaje, basando el análisis en sus distintas características específicas, y al mismo tiempo que considere las especiales características que aporta al proceso la mediación de materiales presentados en formato multimedia e hipermedia, es decir, que sea capaz de dar cuenta de lo específico que añade al proceso la incidencia de las herramientas tecnológicas.

De acuerdo con todo ello, y a continuación, expondremos, en primer lugar, la perspectiva teórica en la que nos situamos para dar cuenta de lo esencial del proceso de

La calidad de los contenidos educativos reutilizables: diseño, usabilidad y prácticas de uso. Mauri, T., Onrubia, J., Coll, C. \& Colomina. 
enseñanza y aprendizaje objeto de análisis, que se vincula, en nuestro caso, a lo que se ha denominado "constructivismo de orientación socio-cultural". En segundo lugar, nos remitiremos a lo específico de este proceso en propuestas formativas que integran contenidos educativos reutilizables. Para ello sostendremos que es necesario identificar lo que de particular añade al proceso de enseñanza y aprendizaje el uso de materiales de este tipo y también la necesidad de situar el análisis de su calidad educativa en el entorno educativo en que estos se integran y que contribuyen a caracterizar. Finalmente, y a partir de estos elementos, plantearemos algunas propuestas sobre las dimensiones más relevantes a utilizar en el análisis de la calidad de propuestas educativas basadas en contenidos educativos reutilizables, así como sobre la necesaria multiplicidad de perspectivas a adoptar en este análisis.

\section{La interactividad y las ayudas educativas como características de los procesos de enseñanza y de aprendizaje}

Sin duda uno de los elementos clave de todo análisis es representarse claramente el objeto del mismo. En este sentido resulta imprescindible hacerse con una representación lo más ajustada posible de la construcción del conocimiento en situaciones de enseñanza y aprendizaje. El constructivismo de orientación sociocultural es una perspectiva teórica específica, capaz de tener en cuenta la naturaleza particular de la construcción del conocimiento y estudiarla en toda su complejidad. Desde esta perspectiva se establece que dicha construcción es un proceso complejo que se lleva a cabo a través de la relación entre tres elementos: el alumno que aprende, desarrollando su actividad mental de carácter constructivo; el contenido objeto de enseñanza y aprendizaje, y el profesor que ayuda al alumno en dicho proceso de construcción, progresando en el grado de significado sobre lo que aprende y siendo progresivamente más capaz de dotarle de sentido. El papel del profesor es el de orientar, guiar y sostener la actividad constructiva del alumno proporcionándole las ayudas educativas necesarias; mediando entre los saberes o contenidos de aprendizaje y la actividad del alumno. Los contenidos, por su parte, mediatizan la actividad conjunta de profesores y alumnos en el proceso de construcción.

En suma, desde la perspectiva establecida, la relación interactiva formada por las relaciones mutuas entre profesor, alumno y contenido o "triángulo interactivo" compone el núcleo básico del análisis del proceso de construcción del conocimiento. Profundizando un poco más en este núcleo, y de modo mucho más específico, podemos llegar a establecer que dicha relación triangular puede concretarse en la interactividad, entendida como la organización de la actividad conjunta, es decir, de las formas que adoptan las actuaciones interrelacionadas de profesores y alumnos en torno a los contenidos y tareas de aprendizaje y de su evolución a lo largo del proceso de construcción del conocimiento.

En consecuencia, para nuestros propósitos lo esencial es constatar que la unidad fundamental del proceso de construcción del conocimiento se concreta en la interactividad. Ello la identifica, a su vez, como la unidad clave del análisis de la calidad de los procesos de enseñanza y de aprendizaje dirigidos a apropiarse de contenidos de aprendizaje determinados. La actividad de mediación del profesor o la ayuda educativa que presta al alumno se convierte en un elemento fundamental e imprescindible del análisis, entendiendo que entre sus características fundamentales está el grado de ajuste

La calidad de los contenidos educativos reutilizables: diseño, usabilidad y prácticas de uso. Mauri, T., Onrubia, J., Coll, C. \& Colomina.

Página 3 de 9 
que ésta consigue a la actividad constructiva del alumno y que se pone de relieve en los intercambios mutuos ente profesor y alumno en relación con los contenidos de aprendizaje. Las ayudas se conciben como de diferentes tipos y se entiende que pueden adquirir una gran variedad de formas concretas, en sintonía con la diversidad de procesos de aprendizaje individuales de cada alumno y con las modificaciones que cada proceso de aprendizaje particular desarrolla lo largo del proceso de enseñanza y aprendizaje.

Así pues, lo esencial del análisis de la calidad educativa es identificar y valorar la diversidad y variedad de formas de ayuda educativa que surgen para ajustar la influencia educativa al alumno, en diversos momentos y ámbitos en que se desarrolla el proceso personal de construcción del conocimiento, acordes con las variaciones que éste pone de manifiesto.

\section{Características de la interactividad en entornos educativos basados en contenidos educativos reutilizables}

La aproximación que acabamos de realizar a la interactividad debe ser completada tomando como referencia algunos elementos característicos de las propuestas formativas que incluyen contenidos educativos reutilizables. En este caso nos detendremos en aquellos que pueden ayudarnos a concretar los niveles clave del análisis que expondremos en la siguiente parte de este escrito.

- La idea de interactividad no se limita a las actuaciones sincrónicas y presenciales de profesor y alumnos.

La interactividad, tal como la hemos definido, incluye la consideración de todas aquellas actuaciones que profesores y alumnos desarrollan sin estar ambos presentes, en un mismo espacio o coincidir en el tiempo. En este sentido, todas aquellas actuaciones de profesores o de alumnos que, aún realizándose sin que dé coincidencia física (espacial y/o temporal), toman sentido únicamente si se las analiza en referencia a las actuaciones anteriores y posteriores de los otros participantes pueden ser consideradas como formando parte de la actividad conjunta o de la interactividad. En el caso de los contenidos educativos reutilizables, se consideran formando parte de la interactividad las actuaciones que un alumno desarrolla individualmente accediendo a la propuesta de contenidos para resolver una tarea de aprendizaje. El profesor o tutor no está presente, pero lo que el alumno realiza tiene relación con lo que ha ocurrido anteriormente en el marco del diseño o la secuenciación de los contenidos incluidos en los materiales y puede, según las condiciones educativas establecidas, tener relación con lo que vaya a ocurrir más tarde, en situaciones de valoración de los resultados de aprendizaje o en las posibilidades establecidas de progreso del alumno.

El análisis de la calidad educativa centrado en la interactividad puede aplicarse adecuadamente al análisis de las propuestas formativas que incluyen contenidos educativos reutilizables. En efecto, a pesar de las apariencias iniciales que sitúan el proceso de construcción del conocimiento en la relación actividad mental del alumno - contenidos, el educador está siempre presente, ya que la actividad del alumno no se explica sin referencias a lo que el profesor o agente educativo haya llevado a cabo para conseguir que el estudiante aprenda. A pesar de todo, una

La calidad de los contenidos educativos reutilizables: diseño, usabilidad y prácticas de uso. Mauri, T., Onrubia, J., Coll, C. \& Colomina. 
afirmación de esta naturaleza necesita ser completada con otras consideraciones que nos proponemos desarrollar en los apartados siguientes.

- La interactividad profesor-alumno - contenido se concreta de modo específico en cada entorno educativo.

Las especiales características de los contenidos educativos reutilizables hacen que dichos materiales puedan integrarse en entornos educativos de características diferentes; por ejemplo, en entornos de autoaprendizaje, semipresenciales y virtuales.

En cada caso, el uso de las TIC puede ser muy diferente y favorecer el acceso a los contenidos contando con ayudas educativas muy variadas. Entre todas ellas no pueden descartarse en absoluto las que se derivan de establecer ámbitos de comunicación entre profesor - alumno mediado por herramientas telemáticas e informáticas; de carácter sincrónico o asincrónico. La interactividad puede variar de uno a otra situación, lo mismo que puede hacerlo su calidad educativa. Ésta puede ser muy diferente, no tanto por los medios o TIC que incluye sino por el uso que se hace de ellos para prestar al alumno ayudas convenientes y adecuadas a sus necesidades educativas.

La importancia de las herramientas y su contribución a la definición de las características de los diferentes entornos hace que resulte pertinente distinguir entre interactividad tecnológica e interactividad pedagógica. La primera se refiere a la incidencia de dichas herramientas y recursos en las formas que toma la relación profesor - alumno -contenidos (es decir, en la estructura de interactividad) y la segunda se refiere a las formas de organización de la actividad conjunta entre profesores y alumnos, y más en concreto a las ayudas educativas que se despliegan en la comunicación entre profesor - alumno en torno a los contenidos y a las tareas de aprendizaje.

- La interactividad en el plano del diseño y del desarrollo de la propuesta formativa que integra contenidos educativos reutilizables.

Al hilo de lo establecido en puntos anteriores, no cabe sino situar el análisis de la interactividad en doble plano: el plano del diseño y el de su desarrollo. Resulta fácil de entender que, aunque en el plano del diseño se hayan previsto diferentes formas de interactividad y de ayudas educativas, no siempre a nivel de desarrollo se procede de acuerdo con las expectativas iniciales. En este sentido, en el análisis de la práctica educativa, no basta con limitarse a uno u otro nivel, sino que es necesario tener en cuenta lo que ambos aportan al proceso para que la interactividad se acabe concretando de un modo determinado.

En consecuencia con todo lo expuesto, la interactividad constituye la unidad básica de análisis de la calidad educativa de una propuesta formativa que incluye contenidos educativos reutilizables en los entornos en que dicha propuesta se integra. Su análisis conlleva tener en cuenta la doble dimensión tecnológica y pedagógica de la interactividad y su concreción tanto en el plano del diseño como en el plano del desarrollo.

\section{Dimensiones para el análisis de la influencia educativa}


De acuerdo con los planteamientos anteriores, proponemos distinguir, al menos, cuatro planos distintos para el análisis de la calidad de propuestas educativas basadas en contenidos educativos reutilizables. Estos cuatro planos surgen del cruce de las dimensiones tecnológica y pedagógica de la interactividad con su doble concreción en términos de diseño y desarrollo de la enseñanza. A su vez, y para cada uno de estos planos, es posible identificar algunas dimensiones específicas de análisis de la calidad de este tipo de propuestas, inspiradas en un modelo de los procesos de enseñanza y aprendizaje de carácter constructivista social.

Estas dimensiones deberían ajustarse en función del tipo de entorno educativo concreto en el cual se lleve a cabo el proceso de enseñanza y aprendizaje: un entorno de autoaprendizaje, un entorno semi-presencial o un entorno virtual. En lo que sigue, nos centramos en su concreción para el caso de entornos virtuales, tal vez los más prototípicamente asociados con el uso de contenidos reutilizables. Los planos y dimensiones de análisis que proponemos serían los siguientes.

1. La "interactividad tecnológica potencial". Este plano remite a las formas de organización de la actividad conjunta, las maneras de estructurar la interactividad por parte de los participantes, que las características y herramientas tecnológicas de la plataforma tecnológica en que se apoya el entorno virtual permiten, promueven, restringen o impiden desarrollar. Entre las dimensiones básicas pertinentes para el análisis de este plano de la interactividad se situarían las siguientes:

- la accesibilidad, fiabilidad y facilidad de uso de la plataforma;

- las herramientas de gestión académica disponibles en la plataforma;

- las herramientas de presentación y acceso a la información disponibles en la plataforma;

- las características tecnológicas de los materiales utilizados para la presentación de la información;

- las herramientas para el diseño de actividades de enseñanza/aprendizaje disponibles en la plataforma;

- las herramientas para el diseño de actividades de evaluación disponibles en la plataforma;

- las herramientas de comunicación disponibles en la plataforma;

- las herramientas para el trabajo colaborativo disponibles en la plataforma;

- las herramientas de seguimiento y evaluación del alumno disponibles en la plataforma;

- los servicios de apoyo accesibles a través de la plataforma.

2. La "interactividad pedagógica potencial". Este plano remite a las formas de organización de la actividad conjunta, las maneras de estructurar la interactividad por parte de los participantes, que las el diseño instruccional previsto para el proceso de enseñanza y aprendizaje en el entorno virtual permite, promueve, restringe o impide desarrollar. Entre las dimensiones básicas pertinentes para el análisis de este plano de la interactividad se situarían las siguientes:

- el modelo psicoeducativo y psicopedagógico general de referencia del diseño instruccional propuesto;

La calidad de los contenidos educativos reutilizables: diseño, usabilidad y prácticas de uso. Mauri, T., Onrubia, J., Coll, C. \& Colomina. 
- los objetivos instruccionales que presiden el diseño propuesto;

- las características de los contenidos que se incluyen en el diseño instruccional propuesto: tipología, organización y secuenciación, formas de presentación:

- las características de los materiales en que se apoya la presentación de los contenidos;

- las actividades de enseñanza y aprendizaje previstas en el diseño instruccional propuesto: tipología, secuencia e interrelación, organización social, tipos de tareas, papel del profesor y los alumnos;

- las actividades de evaluación previstas en el diseño instruccional propuesto: funciones, tipos de tareas, organización social, papel del profesor y los alumnos;

- los recursos didácticos de apoyo previstos en el diseño instruccional propuesto.

3. La “interactividad tecnológica real". Este plano remite a los usos efectivos de las herramientas tecnológicas disponibles en la plataforma que se llevan a cabo en el marco de las formas de organización de la actividad conjunta, la estructura de la interactividad, desarrollada realmente por profesor y alumnos a lo largo del proceso de enseñanza y aprendizaje en el entorno virtual. Entre las dimensiones básicas pertinentes para el análisis de este plano de la interactividad se situarían las siguientes:

- el uso efectivo que realizan profesor y alumnos de las herramientas de gestión académica disponibles en la plataforma;

- el uso efectivo que realizan profesor y alumnos de las herramientas de presentación y acceso a la información disponibles en la plataforma;

- el uso efectivo que realizan profesor y alumnos de las características tecnológicas de los materiales utilizados para la presentación de la información;

- el uso efectivo que realiza el profesor, y eventualmente los alumnos, de las herramientas para el diseño de actividades de enseñanza/aprendizaje disponibles en la plataforma;

- el uso efectivo que realiza el profesor, y eventualmente los alumnos, de las herramientas para el diseño de actividades de evaluación disponibles en la plataforma;

- el uso efectivo que realizan profesor y alumnos de las herramientas de comunicación disponibles en la plataforma;

- el uso efectivo que realizan profesor y alumnos de las herramientas para el trabajo colaborativo disponibles en la plataforma;

- el uso efectivo que realiza el profesor de las herramientas de seguimiento y evaluación del alumno disponibles en la plataforma;

- el uso efectivo que realizan profesor y alumnos de los servicios de apoyo accesibles a través de la plataforma.

4. La "interactividad pedagógica real". Este plano remite a las formas de organización de la actividad conjunta, la estructura de la interactividad, desarrollada realmente por profesor y alumnos a lo largo del proceso de enseñanza y aprendizaje en el entorno virtual como concreción del diseño

La calidad de los contenidos educativos reutilizables: diseño, usabilidad y prácticas de uso. Mauri, T., Onrubia, J., Coll, C. \& Colomina. 
instruccional previsto. Entre las dimensiones básicas pertinentes para el análisis de este plano de la interactividad se situarían las siguientes:

- las actividades de enseñanza y aprendizaje efectivamente desarrolladas por profesor y alumnos a lo largo de la secuencia didáctica;

- la secuencia e interrelación de las actividades de enseñanza y aprendizaje desarrolladas a lo largo de la secuencia didáctica;

- los contenidos que se presentan y trabajan realmente en las distintas actividades de enseñanza y aprendizaje desarrollados a lo largo de la secuencia didáctica y la manera en que se presentan, secuencian y organizan;

- el uso efectivo por parte de profesor y alumnos de los materiales utilizados para la presentación de los contenidos;

- las actividades de evaluación efectivamente desarrolladas por profesor y alumnos a lo largo de la secuencia didáctica;

- el uso efectivo por parte de profesor y alumnos de los recursos didácticos de apoyo previstos en el diseño instruccional propuesto.

En la valoración de estas dimensiones y la concreción de instrumentos de análisis de la calidad de las propuestas educativas resulta fundamental, a nuestro juicio, la consideración de, al menos, tres puntos de vista complementarios: el punto de vista del diseñador, el punto de vista del experto en procesos de enseñanza y aprendizaje (basados en contenidos reutilizables y en el tipo de entorno de que se trate) y el punto de vista del usuario. El diseñador aporta elementos clave sobre los objetivos y referentes del entorno y de la propuesta tecnopedagógica. El experto puede valorar la propuesta educativa tanto desde el punto de vista del diseño como desde el punto de vista del uso. El usuario puede valorar dimensiones de uso no necesariamente coincidentes con las consideradas prioritarias por el diseñador y por el experto. La elaboración de instrumentos de análisis y valoración de la calidad que puedan ser aplicados por estos tres perfiles, y la triangulación de las valoraciones de todos ellos, es esencial, en nuestra opinión, para una adecuada valoración de la calidad de este tipo de propuestas y procesos de enseñanza y aprendizaje.

Fecha de cierre de la redacción del artículo en su primera edición: 12 de febrero de 2005 Aprobación del artículo en su primera edición: 20 de febrero de 2005 Publicación: 15 de julio de 2016

Mauri, T., Onrubia, J., Coll, C. y Colomina, R. (2016). La calidad de los contenidos educativos reutilizables: diseño, usabilidad y prácticas de uso. RED. Revista de Educación a Distancia, 50. Consultado el (dd/mm/aaaa) en http://www.um.es/ead/red/50 


\section{Referencias}

Coll, C. (en prensa) Psicología de la educación y prácticas educativas mediadas por las tecnologías de la información y la comunicación: una mirada constructivista. Sinéctica.

Coll, C. \& Onrubia, J. (1997). The construction of shared meanings in the classroom: joint activity and semiotic devices in the mutual teacher/student control and tracking. In C. Coll \& D. Edwards (Ed.), Teaching, learning and classroom discourse. Approaches to the study of educational discourse (pp. 49-65). Madrid: Fundación Infancia y Aprendizaje.

Coll, C., Colomina, R., Onrubia, J. \& Rochera, M. J. (1992). Actividad conjunta y habla: una aproximación al estudio de los mecanismos de influencia educativa. Infancia y Aprendizaje, 59-60, 189- 232.

EDUS/UOC\&GRINTIE/UB, 2002)2002). Pautas para el análisis de la intervención en entornos de aprendizaje virtual: dimensiones relevantes e instrumentos de evaluación.Informe de progreso presentado al Internet Interdisciplinary Institute (IN3). Barcelona, Diciembre. Disponible en:

http://www.uoc.edu/in3/esp/resultats/documents/edus/informe_EDUS_UB.pdf

\footnotetext{
' Algunas de las ideas recogidas en este texto son tributarias de los resultados teóricos y empíricos obtenidos en el marco del proyecto de investigación, actualmente en curso, "Interacción e influencia educativa: la construcción del conocimiento en entornos electrónicos de enseñanza y aprendizaje" subvencionado por la Comisión Interministerial de Ciencia y Tecnología (proyecto BSO2001-3680-CO20; Investigador principal: César Coll Salvador). Del mismo modo, algunas de las nociones que se recogen en el último apartado del artículo han sido formuladas previamente en un informe de investigación elaborado conjuntamente por los grupos EDUS, de la Universitat Oberta de Catalunya, y GRINTIE, de la Universidad de Barcelona (EDUS/UOC \& GRINTIE/UB, 2002)
}

La calidad de los contenidos educativos reutilizables: diseño, usabilidad y prácticas de uso. Mauri, T., Onrubia, J., Coll, C. \& Colomina. 